\title{
POSITIVITY OF RICCI CURVATURE UNDER THE KÄHLER-RICCI FLOW
}

\author{
DAN KNOPF
}

\begin{abstract}
In each complex dimension $n \geq 2$, we construct complete Kähler manifolds of bounded curvature and nonnegative Ricci curvature whose Kähler-Ricci evolutions immediately acquire Ricci curvature of mixed sign.
\end{abstract}

\section{INTRODUCTION}

In analyzing a geometric evolution equation, it is of critical importance to determine which curvature conditions are preserved or attained under the flow. This is especially true for the Ricci and Kähler-Ricci flows, because of the fundamental ways in which these flows can exploit curvature conditions to reveal topological and canonical geometric properties of their underlying manifolds.

An invariant cone in the space of curvature operators is one that is preserved by a flow. For Ricci flow, the condition $R \geq 0$ is preserved in all dimensions, while the condition $R \leq 0$ is preserved only in real dimension two. Positive curvature operator is preserved in all dimensions [14], but positive sectional curvature is not preserved in dimensions four and above. The known counterexamples are nonproduct metrics on the total space of tangent bundles $\mathbb{R}^{n} \hookrightarrow T \mathcal{S}^{n} \rightarrow \mathcal{S}^{n}$ for $n \geq 2$, constructed recently by $\mathrm{Ni}[22]$. However, positive sectional curvature and positive Ricci curvature are preserved in dimension three [13]. In higher dimensions, Huisken [18] and Nishikawa [23, 24] have demonstrated the invariance of certain sets defined by curvature pinching conditions. Margerin [20] improved Huisken's constant, thereby establishing a sharp invariant set in dimension four. Also in dimension four, H. Chen proved that 2-positivity of the curvature operator is preserved [6], while Hamilton later proved that positive isotropic curvature constitutes an invariant cone [17].

An attractive cone in the space of curvature operators is one that is entered asymptotically at a finite-time singularity. For example, the estimate $R \geq R_{\min }(0)$ implies that the half-space $R \geq 0$ constitutes an attractive cone in all dimensions. Attractive cones place valuable restrictions on which singularity models may appear. Important examples are the pinching estimates for 3-manifolds proved by Ivey [19] and Hamilton [16]. These estimates imply that any rescaled limit formed at a finitetime singularity in dimension three has nonnegative sectional curvature. In turn, this fact is of fundamental importance in Perelman's use of $\kappa$-solutions $[25,26]$ in his recent spectacular progress toward the Geometrization Conjecture.

Date: January 25, 2005; revised March 24, 2005.

2000 Mathematics Subject Classification. Primary 53C44, 53C55; Secondary 53C21.

Keywords. Kähler manifolds, Kähler-Ricci flow, Ricci curvature, invariant and attractive curvature cones.

Author partially supported by NSF grant DMS-0328233. 
For Kähler-Ricci flow, Bando [1] and Mok [21] proved that positive bisectional curvature defines an invariant cone. Positive Ricci curvature is a more problematic condition (except of course in complex dimension one). It is not expected to be an attractive cone. Indeed, certain Kähler-Ricci solitons constructed by Feldman, Ilmanen, and the author [12] have Ricci curvature of mixed sign, yet are strongly conjectured to occur as rescaled limits of singularities encountered by perturbed metrics on the Calabi manifolds $\mathbb{C P}^{1} \hookrightarrow F_{k}^{n} \rightarrow \mathbb{C P}^{n-1}$. (See Example 2.2 of [12].) On the other hand, it would be very useful indeed if positive Ricci curvature constituted an invariant cone for Kähler-Ricci flow. For example, parts of the proofs of some recent important results of X.-X. Chen and Tian for Kähler-Ricci flow [9, 10] only require positive Ricci curvature, but the authors impose the much stronger assumption of positive bisectional curvature to be sure that positive Ricci curvature will be preserved. (These papers also assume the existence of a Kähler-Einstein metric on the underlying manifold. See in particular Remarks 1.5 - 1.7 and Question 9.3 of $[10]$.)

While few might conjecture that positive Ricci curvature is preserved in all complex dimensions, there were until now tantalizing partial results that promised some hope, at least in the critical case of complex dimension two. The history of progress in this area is quite interesting. Some years ago, Cao and Hamilton crafted (but did not publish) a proof that positive orthogonal bisectional curvature $B\left(x, x^{\perp}\right) \geq 0$ is preserved under Kähler-Ricci flow and that its presence ensures that positive Ricci curvature is also preserved, in all dimensions [5]. Recently, Phong and Sturm supplied a public proof that positive Ricci curvature is preserved on a compact complex surface under the stronger hypothesis that the sum of any two eigenvalues of its traceless curvature operator on traceless $(1,1)$-forms is nonnegative [27]. (See also [28].) Later, X.-X. Chen and H. Li generalized the Phong-Sturm result to all dimensions [8]. Most recently, X.-X. Chen announced [7] that the estimate $\mathrm{Rc} \geq \min \left\{0, \mathrm{Rc}_{\min }(0)\right\}$ (along with other results) holds in all dimensions in the presence of positive orthogonal bisectional curvature, thereby strengthening the Cao-Hamilton observation.

The main purpose of this short note is to show that nonnegative Ricci curvature is not preserved for arbitrary complex surfaces. We construct complete Kähler metrics of bounded curvature and nonnegative Ricci curvature whose Kähler-Ricci evolutions immediately acquire Ricci curvature of mixed sign. Let $L_{-1}^{2}$ denote $\mathbb{C}^{2}$ blown up at the origin. (See below for an explanation of the notation.) In this paper, we will prove the following:

Theorem 1. There exist complete Kähler metrics on $L_{-1}^{2}$ of bounded curvature and nonnegative Ricci curvature that immediately develop mixed Ricci curvature when evolved by the Kähler-Ricci flow.

In fact, Theorem 1 is a special case of a more general result. For $n \geq 2$ and $k \in \mathbb{N}$, let $L_{-k}^{n}$ denote the total space of the holomorphic line bundle $\mathbb{C} \hookrightarrow L_{-k}^{n} \rightarrow \mathbb{C P}^{n-1}$ characterized by the equation $\left\langle c_{1},[\Sigma]\right\rangle=-k$, where $c_{1}$ is the first Chern class of the bundle and $\Sigma \approx \mathbb{C P}^{1}$ is a positively oriented generator of $H_{2}\left(\mathbb{C P}^{n-1} ; \mathbb{Z}\right)$. Since the complement of the zero-section of $L_{-k}^{n}$ is biholomorphic to $\left(\mathbb{C}^{n} \backslash\{0\}\right) / \mathbb{Z}_{k}$, one may construct $L_{-k}^{n}$ by gluing a $\mathbb{C P}^{n-1}$ onto $\left(\mathbb{C}^{n} \backslash\{0\}\right) / \mathbb{Z}_{k}$ at the origin. Notice that $L_{-1}^{n}$ is simply the tautological line bundle, i.e. $\mathbb{C}^{n}$ blown up at the origin. 
Theorem 2. For each complex dimension $n \geq 2$ and all integers $k \in[1, n-1]$, there exist Kähler metrics on $L_{-k}^{n}$ that are complete, of bounded curvature, and of nonnegative Ricci curvature, but which immediately develop mixed Ricci curvature when evolved by the Kähler-Ricci flow.

Remark 1. To prove these results, we construct an explicit a one-parameter family (modulo scaling) of Kähler metrics on each bundle $L_{-k}^{n}$.

The remainder of this paper is organized as follows. Since the critical $n=2$ case is most interesting, we begin with a detailed proof of Theorem 1. In Section 2 we provide, for the convenience of the reader, a review of $\mathrm{U}(2)$-invariant metrics on $\mathbb{C}^{2} \backslash\{0\}$. In Section 3, we construct initial metrics on $L_{-1}^{2}$. Then in Section 4 , we finish the proof of Theorem 1. Finally, in Section 5, we provide the straightforward generalizations by which one proves Theorem 2 for all complex dimensions $n \geq 2$.

Acknowledgement 1. The author warmly thanks Huai-Dong Cao for encouraging him to work on this problem.

Question 1. Like Ni's counterexamples for nonnegative sectional curvature [22], the manifolds constructed here are noncompact. Thus it is still an open question of significant interest to find compact counterexamples for which nonnegative Ricci (or sectional) curvatures are not preserved under Kähler-Ricci flow.

Question 2. In either the compact or noncompact case, it is also interesting to ask what are the weakest additional hypotheses under which positive Ricci curvature is preserved.

\section{U(2)-INVARIANT KÄHLER METRICS}

In this section, we review the construction of U(2)-invariant Kähler metrics on $\mathbb{C}^{2} \backslash\{0\}$. Such metrics also appear in $[3,4]$ and [12]. Consider $\mathbb{C}^{2} \backslash\{0\}$ with complex coordinates $\left(z_{1}, z_{2}\right)$. Define

$$
u=\left|z_{1}\right|^{2}, \quad v=\left|z_{2}\right|^{2}, \quad \text { and } \quad w=u+v .
$$

Any function $P: \mathbb{C}^{2} \backslash\{0\} \rightarrow \mathbb{R}$ depending only on $w$ may be written as $P(r)$, where

$$
r=\log w .
$$

Anticipating that we will later abuse notation by regarding $P$ as a function of time as well, we shall use subscripts to denote (partial) derivatives with respect to $r$. Define

$$
\varphi(r)=P_{r}(r) .
$$

Then $P$ is the Kähler potential for a metric $g$ if and only if the derivatives $\varphi$ and $\varphi_{r}$ are everywhere positive. In this case, it is straightforward to compute that

$$
g_{i \bar{\jmath}}=e^{-r} \varphi \delta_{i j}+e^{-2 r}\left(\varphi_{r}-\varphi\right) \bar{z}_{i} z_{j}
$$

and

$$
g^{i \bar{\jmath}}=e^{r} \varphi^{-1} \delta^{i j}+\left(\varphi_{r}^{-1}-\varphi^{-1}\right) z^{i} \bar{z}^{j} .
$$

With respect to the standard bases $\left(d z_{1}, d z_{2}\right)$ and $\left(\partial / \partial z_{1}, \partial / \partial z_{2}\right)$, one can therefore regard $g$ and $g^{-1}$ as the matrices

$$
\left(\begin{array}{ll}
g_{1 \overline{1}} & g_{1 \overline{2}} \\
g_{2 \overline{1}} & g_{2 \overline{2}}
\end{array}\right)=\frac{1}{w^{2}}\left(\begin{array}{cc}
v \varphi+u \varphi_{r} & \left(\varphi_{r}-\varphi\right) \bar{z}_{1} z_{2} \\
\left(\varphi_{r}-\varphi\right) z_{1} \bar{z}_{2} & u \varphi+v \varphi_{r}
\end{array}\right)
$$


and

$$
\left(\begin{array}{ll}
g^{1 \overline{1}} & g^{2 \overline{1}} \\
g^{1 \overline{2}} & g^{2 \overline{2}}
\end{array}\right)=\frac{1}{\varphi \varphi_{r}}\left(\begin{array}{cc}
u \varphi+v \varphi_{r} & \left(\varphi-\varphi_{r}\right) \bar{z}_{1} z_{2} \\
\left(\varphi-\varphi_{r}\right) z_{1} \bar{z}_{2} & v \varphi+u \varphi_{r}
\end{array}\right)
$$

respectively. In the standard coordinate chart, define

$$
G=-\log \operatorname{det} g=2 r-\log \varphi-\log \varphi_{r}
$$

and

$$
\psi=G_{r}=2-\frac{\varphi_{r}}{\varphi}-\frac{\varphi_{r r}}{\varphi_{r}} .
$$

Then the complex Ricci tensor Rc $=R_{i \bar{\jmath}} d z_{i} d \bar{z}_{j}$ may be represented in the same basis as

$$
\left(\begin{array}{ll}
R_{1 \overline{1}} & R_{1 \overline{2}} \\
R_{2 \overline{1}} & R_{2 \overline{2}}
\end{array}\right)=\frac{1}{w^{2}}\left(\begin{array}{cc}
v \psi+u \psi_{r} & \left(\psi_{r}-\psi\right) \bar{z}_{1} z_{2} \\
\left(\psi_{r}-\psi\right) z_{1} \bar{z}_{2} & u \psi+v \psi_{r}
\end{array}\right) .
$$

Abusing terminology slightly, we will say a $(1,0)$-vector $W$ is an eigenvector of the complex Ricci tensor corresponding to the eigenvalue $\lambda$ if $R_{i \bar{\jmath}} W^{i}=\lambda g_{i \bar{\jmath}} W^{i}$. Thus understood, the eigenvalues of Rc are

$$
\lambda_{1}=\frac{\psi}{\varphi} \quad \text { with eigenvector } \quad U=\bar{z}_{2} \frac{\partial}{\partial z_{1}}-\bar{z}_{1} \frac{\partial}{\partial z_{2}}
$$

and

$$
\lambda_{2}=\frac{\psi_{r}}{\varphi_{r}} \quad \text { with eigenvector } \quad V=z_{1} \frac{\partial}{\partial z_{1}}+z_{2} \frac{\partial}{\partial z_{2}} .
$$

The Levi-Civita connection of $g$ is determined by the Christoffel symbols

$$
\begin{aligned}
& \Gamma_{11}^{1}=\frac{\bar{z}_{1}}{w^{2}}\left[u \frac{\varphi_{r r}}{\varphi_{r}}+2 v \frac{\varphi_{r}}{\varphi}+u-2 w\right] \\
& \Gamma_{12}^{1}=\frac{\bar{z}_{2}}{w^{2}}\left[u \frac{\varphi_{r r}}{\varphi_{r}}+(v-u) \frac{\varphi_{r}}{\varphi}-v\right] \\
& \Gamma_{22}^{1}=\frac{z_{1} \bar{z}_{2}^{2}}{w^{2}}\left[\frac{\varphi_{r r}}{\varphi_{r}}-2 \frac{\varphi_{r}}{\varphi}+1\right] \\
& \Gamma_{11}^{2}=\frac{\bar{z}_{1}^{2} z_{2}}{w^{2}}\left[\frac{\varphi_{r r}}{\varphi_{r}}-2 \frac{\varphi_{r}}{\varphi}+1\right] \\
& \Gamma_{12}^{2}=\frac{\bar{z}_{1}}{w^{2}}\left[v \frac{\varphi_{r r}}{\varphi_{r}}+(u-v) \frac{\varphi_{r}}{\varphi}-u\right] \\
& \Gamma_{22}^{2}=\frac{\bar{z}_{2}}{w^{2}}\left[v \frac{\varphi_{r r}}{\varphi_{r}}+2 u \frac{\varphi_{r}}{\varphi}+v-2 w\right]
\end{aligned}
$$

and their conjugates. The complex Riemann tensor is determined by

$$
R_{i \bar{\jmath} k \bar{\ell}}=-g_{m \bar{\ell}} \bar{\partial}_{j} \Gamma_{i k}^{m}=-\partial_{i} \bar{\partial}_{j} g_{k \bar{\ell}}+g^{p \bar{q}} \partial_{i} g_{k \bar{q}} \bar{\partial}_{j} g_{p \bar{\ell}} .
$$

A straightforward computation shows that

$$
\begin{aligned}
R_{i \bar{\jmath} k \bar{\ell}} & =e^{-4 r}\left[-\varphi_{r r r}+4 \varphi_{r r}-2 \varphi_{r}+2 \varphi-4 \frac{\varphi_{r}^{2}}{\varphi}+\frac{\varphi_{r r}^{2}}{\varphi_{r}}\right] \bar{z}_{i} z_{j} \bar{z}_{k} z_{\ell} \\
& +e^{-3 r}\left[-\varphi_{r r}+\varphi_{r}-\varphi+\frac{\varphi_{r}^{2}}{\varphi}\right]\left(\bar{z}_{i} z_{j} \delta_{k \ell}+\bar{z}_{i} \delta_{j k} z_{\ell}+\delta_{i j} \bar{z}_{k} z_{\ell}+\delta_{i \ell} z_{j} \bar{z}_{k}\right) \\
& +e^{-2 r}\left[-\varphi_{r}+\varphi\right]\left(\delta_{i j} \delta_{k \ell}+\delta_{i \ell} \delta_{j k}\right) .
\end{aligned}
$$


To put this into a more useful form, we follow [3]. Since the metric is $\mathrm{U}(2)$-invariant, there is no loss of generality in evaluating the curvature at $(\zeta, 0)$, where $\zeta \neq 0$ is arbitrary. Furthermore, one may assume that the complex tangent space of type $(1,0)$ at $(\zeta, 0)$ is spanned by an orthonormal basis of complex $(1,0)$-vectors $x=x^{i} \partial / \partial z_{i}$ and $y=y^{i} \partial / \partial z_{i}$ such that $x^{2}=0$. Then one can readily compute the bisectional curvatures $B(x, x)=R(x, \bar{x}, x, \bar{x}), B(x, y)=R(x, \bar{x}, y, \bar{y})$, and $B(y, y)=$ $R(y, \bar{y}, y, \bar{y})$ at $(\zeta, 0)$, obtaining

$$
\begin{aligned}
\left.B(x, x)\right|_{(\zeta, 0)} & =\frac{1}{|\zeta|^{4}}\left(\frac{\varphi_{r r}^{2}}{\varphi_{r}}-\varphi_{r r r}\right)\left|x^{1}\right|^{4} \\
\left.B(x, y)\right|_{(\zeta, 0)} & =\frac{1}{|\zeta|^{4}}\left[\left(\frac{\varphi_{r r}^{2}}{\varphi_{r}}-\varphi_{r r r}\right)\left|x^{1}\right|^{2}\left|y^{1}\right|^{2}+\left(\frac{\varphi_{r}^{2}}{\varphi}-\varphi_{r r}\right)\left|x^{1}\right|^{2}\left|y^{2}\right|^{2}\right] \\
\left.B(y, y)\right|_{(\zeta, 0)} & =\frac{1}{|\zeta|^{4}}\left[\left(\frac{\varphi_{r r}^{2}}{\varphi_{r}}-\varphi_{r r r}\right)\left|y^{1}\right|^{4}+4\left(\frac{\varphi_{r}^{2}}{\varphi}-\varphi_{r r}\right)\left|y^{1}\right|^{2}\left|y^{2}\right|^{2}+2\left(\varphi-\varphi_{r}\right)\left|y^{2}\right|^{4}\right] .
\end{aligned}
$$

Observe in particular that the choices

$$
\left.X\right|_{(\zeta, 0)}=\frac{|\zeta|}{\sqrt{\varphi_{r}}} \frac{\partial}{\partial z_{1}} \quad \text { and }\left.\quad Y\right|_{(\zeta, 0)}=\frac{|\zeta|}{\sqrt{\varphi}} \frac{\partial}{\partial z_{2}}
$$

allow one to recover (2.6) in the form

$$
\begin{aligned}
\left.\operatorname{Rc}(X, \bar{X})\right|_{(\zeta, 0)} & =B(X, X)+B(X, Y)=\frac{\psi_{r}}{\varphi_{r}} \\
\left.\operatorname{Rc}(Y, \bar{Y})\right|_{(\zeta, 0)} & =B(Y, Y)+B(X, Y)=\frac{\psi}{\varphi} .
\end{aligned}
$$

\section{An initial metric of nonnegative Ricci Curvature}

We now want to investigate whether there exists a U(2)-invariant Kähler potential $P$ on $\mathbb{C}^{2} \backslash\{0\}$ with the following global properties:

(1) $\varphi>0$ everywhere;

(2) $\varphi_{r}>0$ everywhere;

(3) $\psi>0$ everywhere;

(4) $\psi_{r} \geq 0$ everywhere;

(5) $g$ has bounded curvature;

(6) $g$ extends smoothly to a complete metric as $r \rightarrow+\infty$; and

(7) $g$ extends smoothly to a complete metric as $r \rightarrow-\infty$.

Furthermore, we require that there exists at least one point $(\zeta, 0) \in \mathbb{C}^{2} \backslash\{0\}$ such that with respect to an orthonormal basis $x, y$ for the complex tangent space of type $(1,0)$ at $(\zeta, 0)$, the following local properties hold:

I $B(x, y)<0$; and

II $B(x, x)=-B(x, y)$.

It may seem foolhardy to expect a single function $P$ to satisfy all nine of these requirements. However, after some study, one is led to the following Ansatz: let $a>0$ be a constant to be determined and consider the second-order ODE

$$
\left[\log \left(\varphi \varphi_{r}\right)\right]_{r}=a .
$$


Its general solution is $\sqrt{2 e^{a r+b} / a+c}$, where $b$ and $c$ are arbitrary. Without loss of generality, one may eliminate $b$ by scaling, yielding

$$
\varphi(r)=\sqrt{\frac{2}{a} e^{a r}+c .}
$$

One then has

$$
\varphi \varphi_{r}=e^{a r}
$$

so that global properties (1) and (2) are satisfied whenever $c \geq 0$. Moreover,

$$
\psi=2-a
$$

everywhere. So properties (3) and (4) are satisfied whenever $a \in(0,2)$.

To evaluate the bisectional curvatures of the resulting metric, it suffices to consider the orthonormal basis $(2.7)$ for the complex tangent space of type $(1,0)$ at $(\zeta, 0)$, where $\zeta \neq 0$ is arbitrary. One finds that

$$
B(X, X)=\frac{a c}{\varphi^{3}}=-B(X, Y),
$$

so that local properties I and II will in fact be satisfied globally if $c>0$. The choices $a \in(0,2)$ and $c>0$ also ensure that

$$
B(Y, Y)=\frac{2}{\varphi^{3}}\left[\left(\frac{2}{a}-1\right) e^{a r}+c\right]>B(X, X) .
$$

Because $\varphi(r) \rightarrow \sqrt{c}>0$ as $r \rightarrow-\infty$ and $\varphi(r)=\mathcal{O}\left(e^{(a / 2) r}\right)$ as $r \rightarrow \infty$, the curvatures are globally bounded, satisfying property (5). To investigate completeness at spatial infinity, observe that (3.3) implies that $\varphi_{r}=\mathcal{O}\left(e^{a r}\right)$ as $r \rightarrow-\infty$ and that $\varphi_{r}=\mathcal{O}\left(e^{(a / 2) r}\right)$ as $r \rightarrow \infty$. Since radial paths emanating from the origin are geodesics and the length of such a path is $\int_{-\infty}^{\infty} \sqrt{\varphi_{r}} d r$, it is easy to see that the metric is complete as $r \rightarrow+\infty$, satisfying global property (6).

On the other hand, the metric distance from an arbitrary point to $r=-\infty$ is finite. Our choice of $c>0$ makes it impossible to complete the metric smoothly by gluing in a point at the origin. But Calabi's theorem [2] tells us that $g$ will extend to a smooth Kähler metric on $L_{-k}^{2}$ if $\varphi$ has an asymptotic expansion near $w=0$ of the form

$$
\varphi=a_{0}+a_{1} w^{k}+a_{2} w^{2 k}+\mathcal{O}\left(w^{3 k}\right)
$$

with $a_{0}$ and $a_{1}$ both positive. (See Section 4.1 of [12] for an exposition.) The function (3.2) will have such an expansion if $a$ is a positive integer. Fortunately for us, there is a (unique) positive integer in the interval $(0,2)$. Taking $a=1$ thus lets us satisfy global property (7). Notice that for each $c>0$, the solution $\varphi$ then admits the expansion

$$
\varphi=\sqrt{c}+\frac{1}{\sqrt{c}} w-\frac{1}{2 c^{3 / 2}} w^{2}+\cdots .
$$

In particular, $2 \pi \sqrt{c}$ is the area of the $\mathbb{C P}^{1}$ that constitutes the zero-section of the bundle.

Remark 2. The Kähler manifolds $\left(L_{-1}^{2}, g\right)$ we have constructed are asymptotic at infinity to a Kähler cone $\Gamma_{1}$ on $\mathbb{C}^{2} \backslash\{0\}$ whose cone angle around the origin in each complex line is $\pi$ and whose density ratio is $\operatorname{Vol}_{\Gamma_{1}}\left(B_{1}\right) / \operatorname{Vol}_{\mathbb{C}^{2}}\left(B_{1}\right)=1 / 4$. 


\section{EfFect of the KäHLER-RicCI FLOW}

We now consider the Kähler-Ricci evolution $\left(L_{-1}^{2}, g(t)\right)$, where we take $P(r, 0)$ to be the Kähler potential $P(r)$ constructed above. Again choose an arbitrary point $(\zeta, 0) \in \mathbb{C}^{2} \backslash\{0\}$. Because

$$
\left.g\right|_{(\zeta, 0)}=\frac{1}{|\zeta|^{2}}\left(\begin{array}{cc}
\varphi_{r} & 0 \\
0 & \varphi
\end{array}\right)
$$

and

$$
\left.\operatorname{Rc}\right|_{(\zeta, 0)}=\frac{1}{|\zeta|^{2}}\left(\begin{array}{cc}
\psi_{r} & 0 \\
0 & \psi
\end{array}\right)
$$

in the standard basis, the Kähler-Ricci flow

$$
\frac{\partial}{\partial t} g=-\mathrm{Rc}
$$

will be satisfied if and only if $\varphi$ evolves by the $\operatorname{PDE} \varphi_{t}=-\psi$; to wit,

$$
\varphi_{t}=\frac{\varphi_{r r}}{\varphi_{r}}+\frac{\varphi_{r}}{\varphi}-2
$$

Since $\partial / \partial r$ and $\partial / \partial t$ commute, it follows that the Ricci potential $\psi$ must evolve by the PDE

$$
\psi_{t}=\frac{\psi_{r r}}{\varphi_{r}}-\frac{\varphi_{r r} \psi_{r}}{\varphi_{r}^{2}}+\frac{\psi_{r}}{\varphi}-\frac{\varphi_{r} \psi}{\varphi^{2}}
$$

Since $\psi(\cdot, 0) \equiv 1$, this reduces to

$$
\left.\frac{\partial}{\partial t} \psi\right|_{t=0}=-\frac{\varphi_{r}}{\varphi^{2}}
$$

at the initial time. A further computation then shows that

$$
\left.\frac{\partial}{\partial t} \psi_{r}\right|_{t=0}=\frac{e^{r}}{\varphi^{5}}\left(e^{r}-c\right)
$$

which is strictly negative for all $r<\log c$. Since $\psi_{r}(\cdot, 0) \equiv 0$, the complex Ricci tensor must acquire a negative eigenvalue $\lambda_{2}<0$ at all such points for small times $t>0$. This completes the proof of Theorem 1 .

\section{The general Case: Complex dimension $n \geq 2$}

If $P$ is a $\mathrm{U}(n)$-invariant Kähler potential on $\mathbb{C}^{n} \backslash\{0\}$, formulas (2.2) for $g$ and (2.3) for $g^{-1}$ remain unchanged. Formula (2.4) becomes

$$
G=n r-\log \varphi-\log \varphi_{r}
$$

whence (2.5) is replaced by

$$
\psi=G_{r}=n-\frac{\varphi_{r}}{\varphi}-\frac{\varphi_{r r}}{\varphi_{r}}
$$

One calculates easily that

$$
R_{i \bar{\jmath}}=e^{-r} \psi \delta_{i j}+e^{-2 r}\left(\psi_{r}-\psi\right) \bar{z}_{i} z_{j} .
$$


To display the eigenvalues of Rc, one may without loss of generality fix $Z=$ $(\zeta, 0, \ldots, 0) \in \mathbb{C}^{n} \backslash\{0\}$ with $\zeta \neq 0$ arbitrary. Then

$$
\left.g\right|_{Z}=\frac{1}{|\zeta|^{2}}\left(\begin{array}{cccc}
\varphi_{r} & & & \\
& \varphi & & \\
& & \ddots & \\
& & & \varphi
\end{array}\right)
$$

and

$$
\left.\operatorname{Rc}\right|_{Z}=\frac{1}{|\zeta|^{2}}\left(\begin{array}{cccc}
\psi_{r} & & & \\
& \psi & & \\
& & \ddots & \\
& & & \psi
\end{array}\right) \text {. }
$$

If $\varphi$ is defined by (3.2) with $a>0$, one finds that

$$
\psi \equiv n-a,
$$

hence that $\psi_{r} \equiv 0$. Equation (4.1) becomes

$$
\varphi_{t}=\frac{\varphi_{r r}}{\varphi_{r}}+\frac{\varphi_{r}}{\varphi}-n
$$

while (4.2) remains unchanged. The analog of (4.3) is

$$
\left.\frac{\partial}{\partial t} \psi_{r}\right|_{t=0}=(n-a) \frac{e^{a r}}{\varphi^{5}}\left(e^{a r}-a c\right)
$$

which is strictly negative for all $a \in(0, n)$ and $c>0$ whenever $r<\frac{1}{a} \log (a c)$. If we take $a \in(0, n)$ to be an integer, then $\varphi$ will have an asymptotic expansion near the origin of the form

$$
\varphi=\sqrt{c}+\frac{1}{a \sqrt{c}} w^{a}-\frac{1}{2 a^{2} c^{3 / 2}} w^{2 a}+\cdots .
$$

Hence by Calabi's theorem, $g$ will extend to a smooth metric on $L_{-a}^{n}$ for all such $a$. It is easy to check that $g$ is complete and asymptotic to a Kähler cone at infinity, hence of bounded curvature. This completes the proof of Theorem 2 .

Remark 3. For each $1 \leq k \leq n-1$, the Kähler manifolds $\left(L_{-k}^{n}, g\right)$ we have constructed are asymptotic at infinity to a Kähler cone $\Gamma_{k}$ on $\mathbb{C}^{n} \backslash\{0\}$ whose cone angle at the origin is $\pi$ and whose density ratio is $\operatorname{Vol}_{\Gamma_{k}}\left(B_{1}\right) / \operatorname{Vol}_{\mathbb{C}^{n}}\left(B_{1}\right)=k^{1-n} / 2^{n}$.

\section{REFERENCES}

[1] Bando, Shigetoshi. On the classification of three-dimensional compact Kaehler manifolds of nonnegative bisectional curvature. J. Differential Geom. 19 (1984), no. 2, 283-297.

[2] Calabi,Eugenio. Extremal Kähler metrics. Seminar on Differential Geometry, 259-290, Ann. of Math. Stud., 102, Princeton Univ. Press, Princeton, N.J., 1982.

[3] Cao, Huai-Dong. Existence of gradient Kähler-Ricci solitons. Elliptic and parabolic methods in geometry (Minneapolis, MN, 1994), 1-16, A K Peters, Wellesley, MA, 1996.

[4] Cao, Huai-Dong. Limits of solutions to the Kähler-Ricci flow. J. Differential Geom. 45 (1997), no. 2, 257-272.

[5] Cao, Huai-Dong; Hamilton, Richard S. On the preservation of positive orthogonal bisectional curvature under the Kähler-Ricci flow. (1988) Private communication (unpublished).

[6] Chen, Haiwen. Pointwise $\frac{1}{4}$-pinched 4-manifolds. Ann. Global Anal. Geom. 9 (1991), no. 2, 161-176.

[7] Chen, Xiuxiong. Private communication. 
[8] Chen, Xiuxiong; Li, H. The Kähler-Ricci flow on Kähler manifolds with 2 traceless bisectional curvature operator. arXiv:math.DG/0503645.

[9] Chen, Xiuxiong; Tian, Gang. Ricci flow on Kähler-Einstein surfaces. Invent Math. 147 (2002), no. 3, 487-544.

[10] Chen, Xiuxiong; Tian, Gang. Ricci flow on Kähler-Einstein manifolds. arXiv:math. $\mathrm{DG} / 0108179$.

[11] Chow, Bennett. The Ricci flow on the 2-sphere. J. Differential Geom. 33 (1991), no. 2, $325-334$.

[12] Feldman, Mikhail; Ilmanen, Tom; Knopf, Dan. Rotationally symmetric shrinking and expanding gradient Kähler-Ricci solitons. J. Differential Geom. 65 (2003), no. 2, 169-209.

[13] Hamilton, Richard S. Three-manifolds with positive Ricci curvature. J. Differential Geom. 17 (1982), no. 2, 255-306.

[14] Hamilton, Richard S. Four-manifolds with positive curvature operator. J. Differential Geom. 24 (1986), no. 2, 153-179.

[15] Hamilton, Richard S. The Ricci flow on surfaces. Mathematics and general relativity (Santa Cruz, CA, 1986), 237-262, Contemp. Math., 71, Amer. Math. Soc., Providence, RI, 1988 .

[16] Hamilton, Richard S. The formation of singularities in the Ricci flow. Surveys in differential geometry, Vol. II (Cambridge, MA, 1993), 7-136, Internat. Press, Cambridge, MA, 1995.

[17] Hamilton, Richard S. Four-manifolds with positive isotropic curvature. Comm. Anal. Geom. 5 (1997), no. 1, 1-92.

[18] Huisken, Gerhard. Ricci deformation of the metric on a Riemannian manifold. J. Differential Geom. 21 (1985), no. 1, 47-62.

[19] Ivey, Thomas. Ricci solitons on compact three-manifolds. Differential Geom. Appl. 3 (1993), no. 4, 301-307.

[20] Margerin, Christophe. A sharp characterization of the smooth 4-sphere in curvature terms. Comm. Anal. Geom. 6 (1998), no. 1, 21-65.

[21] Mok, Ngaiming. The uniformization theorem for compact Kähler manifolds of nonnegative holomorphic bisectional curvature. J. Differential Geom. 27 (1988), no. 2, 179-214.

[22] Ni, Lei. Ricci flow and nonnegativity of curvature. arXiv:math.DG/0305246.

[23] Nishikawa, Seiki. Deformation of Riemannian metrics and manifolds with bounded curvature ratios. Geometric measure theory and the calculus of variations (Arcata, Calif., 1984), 343-352, Proc. Sympos. Pure Math., 44, Amer. Math. Soc., Providence, RI, 1986.

[24] Nishikawa, Seiki. On deformation of Riemannian metrics and manifolds with positive curvature operator. Curvature and topology of Riemannian manifolds (Katata, 1985), 202-211, Lecture Notes in Math., 1201, Springer, Berlin, 1986.

[25] Perelman, Grisha. The entropy formula for the Ricci flow and its geometric applications. arXiv:math.DG/0211159.

[26] Perelman, Grisha. Ricci flow with surgery on three-manifolds. arXiv:math.DG/0303109.

[27] Phong, Duong H.; Sturm, Jacob. On the Kähler-Ricci flow on complex surfaces. arXiv: math.DG/0407232.

[28] Phong, Duong H.; Sturm, Jacob. On stability and the convergence of the Kähler-Ricci flow. arXiv:math.DG/0412185.

University of TeXas at Austin

E-mail address: danknopf@math.utexas.edu

$U R L:$ http://www.ma.utexas.edu/ ${ }^{\sim}$ danknopf/ 